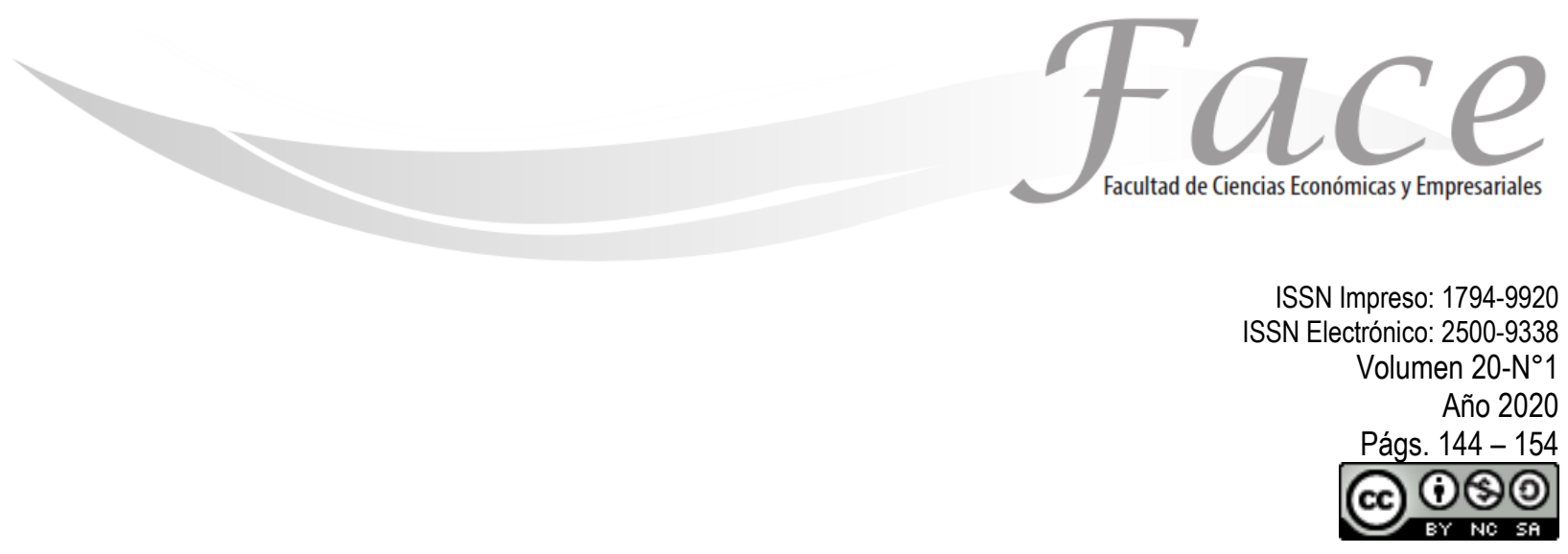

\title{
GOBERNANZA 4.0: DESAFÍOS EN LA INNOVACIÓN EDUCACIÓN SUPERIOR EN LOS PROGRAMAS EDUCATIVOS DE TURISMO Y GASTRONOMÍA
}

\author{
Mtra. Angélica Ruth Terrazas Juárez* \\ ENLACE ORCID: https://orcid.org/0000-0001-6822-528x. \\ Dra. Erika Cruz Coria** \\ ENLACE ORCID: https://orcid.org/0000-0001-7984-0069 \\ Dr. Pedro Alfonso Ramos Sánchez ${ }^{* * *}$ \\ ENLACE ORCID: https://orcid.org/0000-0002-2156-3553
}

Fecha de Recepción: Septiembre 5 de 2020

Fecha de Aprobación: Diciembre 14 de 2020

\section{Resumen:}

La presente investigación analiza las nuevas formas de gobernanza en la Educación Superior (ES) promovidas a partir de los cambios en la industria 4.0, en el sector turístico que fue afectado por la pandemia del COVID-19. Este trabajo se sustenta en una revisión documental sobre la industria 4.0 y su relación con la gobernanza 4.0, permitiendo definir un marco de referencia para determinar las nuevas formas de gobierno en la ES, identificando los cambios disruptivos de este siglo; lo que permitió establecer una dinámica en adoptar políticas públicas hacia la gobernanza no presencial.

Palabras Clave: gobernanza, innovación, Educación Superior, turismo, gastronomía

\footnotetext{
* Maestra en Administración de Recursos Humanos. Profesora Investigadora del Instituto de Ciencias Económico Administrativas, perteneciente a la Universidad Autónoma del Estado de Hidalgo, México. Contacto: tjuarez@uaeh.edu.mx

** Doctor a en Ciencias Ambientales Profesora Investigadora del Instituto de Ciencias Económico Administrativas, perteneciente a la Universidad Autónoma del Estado de Hidalgo, México. Contacto: erika_cruz10093@uaeh.edu.mx

*** Doctor en Desarrollo Económico y Sectorial Estratégico. Profesor Investigador del Instituto de Ciencias Económico Administrativas, perteneciente a la Universidad Autónoma del Estado de Hidalgo, México. Contacto: pedro ramos3944@uaeh.edu.mx
} 


\title{
GOVERNANCE4.0: CHALLENGES IN HIGHER EDUCATION INNOVATION \\ IN TOURISM AND GASTRONOMY EDUCATIONAL PROGRAMS
}

\begin{abstract}
:
This research analyzes the new forms of governance in higher education (HE) promoted from industry 4.0., mainly in the tourism industry which were the sectors with the greatest crisis due to the pandemic of the Covid-19. This paper is based on a documentary review on industry 4.0 and its relation with governance 4.0; this allowed defining a reference framework to determine the new forms of governance management in the HE identifying the disruptive changes of this century. This study shows the dynamic of generating public policy towards governance in a remote.
\end{abstract}

Keywords: governance, Innovation, Higher Education, tourism, gastronomy

\section{GOVERNANÇA 4.0: DESAFIOS NA EDUCAÇÃO SUPERIOR INOVAÇÃO NOS PROGRAMAS EDUCACIONAIS DE TURISMO E GASTRONOMIA}

\begin{abstract}
Resumo:
Esta pesquisa analisa as novas formas de governança no ensino superior (ES) promovidas a partir da indústria 4.0, principalmente na indústria do turismo que foram os setores com maior crise devido à pandemia do Covid-19. Este artigo é baseado em uma revisão documental sobre a indústria 4.0 e sua relação com a governança 4.0; isso permitiu definir um quadro de referência para determinar as novas formas de gestão de governança na ES identificando as mudanças disruptivas deste século. Este estudo mostra a dinâmica de geração de políticas públicas voltadas para a governança a distância.

Palavras-chave: governança, inovação, ensino superior, turismo, gastronomia.
\end{abstract}




\section{INTRODUCCIÓN:}

En el marco de los grandes cambios nacionales e internacionales, la educación se ha transformado de manera radical, y esto conlleva igualmente a cambios demográficos, económicos, sociales, políticos y ahora por la emergencia sanitaria, se tuvo que establecer estrategias para evitar la propagación de este virus, la cual una de ellas fue que los gobiernos determinaron en suspender actividades escolares presenciales en todos los niveles escolares, aplicando diversas acciones en utilizar herramientas didácticas para poder culminar el primer semestre del ciclo escolar 2020 cuya solución propuesta fue la de implementar y en su caso, el de fortalecer el uso de diversas tecnologías digitales para realizar de forma remota, a distancia, o virtual.

En el caso de las universidades, se obligó a garantizar el desarrollo de sus funciones a través de la mediación de tecnologías sincrónicas y asincrónicas, lo que esta modalidad virtual produjo una aceleración de sus modelos de gobernanza hacia la promoción de la autonomía, el trabajo por objetivos, colaborativo y en red, impulsándose la alfabetización digital de toda la comunidad universitaria, dando pauta hacia la migración de una universidad tradicional hacia una emergencia digital lo que se tuvo que establecer la capacitación de la comunidad universitaria en el uso de herramientas digitales, así como por las asimetrías de conectividad, de contexto socioeconómico y de gestión de la autonomía, cuestiones que quedaron evidenciadas frente al aislamiento social obligatorio (De Vicenzi, 2020).

En ese sentido, la sociedad del conocimiento desafía a las instituciones de educación superior (IES) a tomar decisiones y a asumir nuevos liderazgos en la conducción de sus proyectos institucionales a través de modelos educativos alternativos. Esto supone rediseñar estructuras y formas de gobierno para aumentar sus capacidades estratégicas frente al medio y a los actores sociales externos, siendo la cuestión de la gobernanza un factor clave (Brunner, 2011).
Por lo tanto el presente trabajo, explora ese componente ideacional de la gobernanza de las IES, en términos metodológicos en la de extender el uso de una teoría existente a una nueva evidencia empírica donde se manifiesta el poder ideacional (Carstensen y Schmidt, 2016), es decir ejercer el poder a través y sobre de las ideas, en relación con las principales dimensiones de la gobernanza de los sistemas nacionales de las IES para la próxima tercera década del siglo XXI.

\section{MARCO TEÓRICO:}

\subsection{La universidad como elemento de una nueva gobernanza hacia la innovación}

La Universidad, como institución, construye configuraciones culturales informales y compartidas, símbolos y esquemas cognitivos asumidos como verdaderos, así como sistemas de reglas formales. Establece rutinas y procesos que sostienen estas configuraciones, símbolos y reglas, garantizando su reproducción a lo largo del tiempo (Campbell, 2009), lo que permiten pensar a la Universidad como una estructura en donde convive la especialización del conocimiento, una cultura institucional integradora a partir del establecimiento de una misión y un proyecto institucional compartido por todos los actores que la conforman que se articulan a partir de un sistema de creencias definido como la cultura institucional reflejada en su diseño organizacional que debe guardar coherencia con los objetivos y propósitos de la institución, con el contexto social en el cual se inserta y con las regulaciones del Estado (Miceli, 2019), y que deben enfrentar a un proceso denominado isomorfismo institucional, en la que las organizaciones buscan alcanzar su legitimidad frente a los distintos agentes externos a partir de la competencia por recursos y la calidad de sus programas, pero también, por otros factores 
vinculados con el poder político, la legitimidad institucional y por favorables condiciones sociales y económicas (Dimaggio y Powell,1983), lo que podrían conducir hacia un modelo único e ideal de universidad, que va dando forma y homogeneizando al conjunto del sistema que debe incluir ciertos elementos, tales como: la legitimidad de las autoridades como resultado de la capacidad de representación de los distintos grupos que integran la comunidad académica; las condiciones institucionales o reglas del juego que dan lugar a las relaciones de cooperación entre los actores y generan el diseño institucional de gobernanza, y el tercer elemento la sociedad que en conjunto se le denomina la triple hélice como promotora de la innovación en las sociedades mediante la creación de capacidades y alianzas estratégicas (Castillo Hernández, Lavín Verástegui y Pedraza Melo, 2014; Salazar y Valderrama, 2010) y que ha sido validada desde diferentes perspectivas acorde a los intereses de investigación.

\subsection{Gobernanza y Gobernanza 4.0}

El término gobernanza presenta distintas aristas y dimensiones el cuál pasó de referirse a la acción del gobierno o ejercicio de gobierno en una región (Loyo, 2002; citado por Mayorga y Córdova, 2007) a considerarse a un marco de reglas, instituciones y prácticas establecidas que sientan los límites y los incentivos para el comportamiento de los individuos, las organizaciones y las empresas (Prats, 2001; en Mayorga y Córdova, 2007). Según Mayntz (2001; citado en Lecay, 2006), la gobernanza requiere de las siguientes condiciones para su desarrollo: poder distribuido, disposición y administración de recursos; la existencia de una sociedad civil fuerte y organizada, además de un fuerte compromiso cívico y un considerable nivel educativo. El compromiso de la sociedad para compartir las acciones de gobierno, solo es posible si la sociedad ha desarrollado, a través de sus procesos educativos una conciencia cívica sólida y un compromiso ciudadano.

En América Latina, la práctica de la gobernanza es incipiente, la deficiencia del sistema educativo, la exclusión y el rezago educativo genera dependencia del gobierno. Los espacios de discusión aún son escasos. La educación en América Latina se considera un derecho de la sociedad y es un servicio que es brindado primordialmente por el Estado, por ésta razón la gobernanza de los sistemas educativos se convierte en una tarea fundamental de los gobiernos, debido a su gestión y sus relaciones con entidades y actores externos para asegurar los objetivos de la educación superior (Brunner, 2011).

La gobernanza está relacionada con las estructuras de toma de decisiones, sus procesos y sus objetivos, pero también con la forma de liderazgo y la estructura administrativa que adopta una institución, a nuevas relaciones entre distintos actores: las instituciones de educación superior, el Estado o autoridades públicas y la sociedad (Kheim, 2012). Actualmente, la Gobernanza 4.0 es una modalidad de gobierno y gestión que busca adaptarse a las nuevas revolución industrial, en la que las nuevas tecnologías difuminan los límites físicos, digitales y biológicos (Schwab, 2016).

Un punto de importancia es que, más allá de las características que asume una gobernanza favorable, el gobierno y la gestión de una IES se sustenta en la existencia o inexistencia de estructuras que permiten el logro de los fines y propósitos establecidos en su misión institucional y con miras para abordar todo lo que atañe al futuro del trabajo con el fin de preverse que la disrupción tecnológica interactuará con otras variables socioeconómicas, geopolíticas y demográficas, generando una gran convulsión en el mercado laboral (Parker, 2015), y por lo tanto esto puede apuntar que años venideros la destrucción de profesiones va a ser mayor que la capacidad de nuestra sociedad para crear otras nuevas, generando un incremento de tasas de desempleo lo que habrá dificultades para cubrir ciertos puestos de trabajo y buena parte del actual "saber" y "saber hacer" requerido a los profesionales se está requiriendo completar con su "saber estar" y "saber ser" 
(Echeverría, 2016), lo que será prioritario retener a los mejores talentos y en la mayoría de ellas el impacto de la tecnología acortará la vigencia de las competencias, que necesitarán actualizarlas a lo largo de toda la vida, lo que estarán inmersos en la cuarta revolución industrial, los empleadores no pueden seguir siendo meros consumidores pasivos de trabajadores competentes, los políticos deben liderar profundos cambios en el sistema educativo y en la regulación del mercado de trabajo y la ciudadanía ha de involucrarse en procesos de aprendizaje a lo largo del tiempo (Raina, 2016).

\subsection{Las competencias que se identifican en base a los cambios disruptivos del siglo veintiuno}

En la Cumbre de Davos de 2016, se comentó que: "La cuarta revolución industrial, abarca avances en áreas un tanto inconexas como inteligencia artificial $y$ aprendizaje automático, robótica, nanotecnología, genética, impresión 3-D, y biotecnología, provocando en los próximos cinco años una transformación generalizada no solo de los modelos de negocio, sino también de los mercados de trabajo, con enormes cambios previstos en el conjunto de competencias necesarias para prosperar en el nuevo escenario" (WEF, 2016:5), afectando como individuos en múltiples facetas nuestras vidas como, la longevidad, la salud, el sentido de la privacidad, los procesos cognitivos, la manera de relacionarnos con los demás, el tiempo dedicado al trabajo y al ocio; así como el desarrollo de las carreras profesionales (Kaye, Williams \& Cowart, 2017).

Mientras las tres primeras revoluciones impactaron primordialmente en maquinaria y tecnología dura, en esta cuarta, las personas serán la piedra angular de esta gran transformación, lo que obliga a afrontar individual y colectivamente los retos planteados, lo que implica la disposición al cambio de las personas, de actores políticos, de los sectores públicos y privados; la academia, empresas, sindicatos, así como de la sociedad en general, aunado a ello es preciso reformar las organizaciones e instituciones establecidas, comprometerse con los nuevos actores emergentes y apoyar a los ciudadanos, cuyas vidas pueden verse afectadas por los cambios que se avecinan (Neufeind, Reilly \& Ranft, 2018), esto conlleva a que se generarán nuevas competencias que se identifican en base a los cambios disruptivos del siglo veintiuno y cómo se afrentarán en las futuras disciplinas y que implicarán al esquema personas, empresas, gobierno, universidades, siendo las competencias claves que se van a relacionar con el trabajo futuro.

Considerando lo anterior esto implicará tanto para las personas y empleadores, como para los sistemas educativos, que, a juicio de sus autores, necesitarán ante todo adaptarse a las necesidades cambiantes de la sociedad y planificar para un futuro de 20 a 30 años. Por una parte, han de propiciar una mayor comprensión, mejor práctica del proceso de enseñanza-aprendizaje y de evaluación, acorde con las competencias más demandadas. $Y$ por la otra, ofrecer al alumnado itinerarios flexibles y adaptados a sus diversas demandas de desarrollo y acreditación de sus competencias. $Y$ para todo ello es imprescindible contar con un profesorado incentivado de modo eficiente y eficaz, bien formado y en actitud de reciclaje permanente, para que puedan dar respuesta a los requerimientos de la sociedad del siglo $X X I$.

\subsection{La migración de la universidad 2.0 a una universidad 4.0}

Los grandes cambios que vemos con las tecnologías digitales están volviendo obsoletos los enfoques tradicionales de la pedagogía clásica y sus titulaciones convencionales. Algunas habilidades blandas que pronto serán indispensables incluyen el pensamiento cognitivo de orden superior, pensamiento adaptativo innovador, gestión de la carga cognitiva, múltiples alfabetismos, solución de situaciones complejas, 
habilidades sociales, destrezas elásticas y competencias transvergentes para realizar tareas de naturaleza cambiante. Las competencias digitales también provocarán diversidad de conexiones sinápticas y sociales que se volverán cada vez más flexibles y adaptables (Barnett, 2004). Por lo que el autor considera que la evolución de una Universidad 2.0, en la cual fue considerada la universidad de investigación surgió en las sociedades posindustriales, por lo que se convirtieron en el punto focal para el avance tecnológico impulsado por la investigación, basada en la masificación de la educación con el profesor como el principal proveedor de conocimiento y el alumno como receptor pasivo que absorbe los contenidos; mientras que la Universidad 3.0 se concebía como la universidad emprendedora, basada en la integración de las computadoras e Internet en la enseñanza y el aprendizaje, lo que ayudó a aumentar el acceso y equidad educativa, bajo esta evolución, la universidad 4.0 refiere a una universidad ecológica orientada hacia el exterior, profundamente conectada con la industria y las comunidades a su alrededor, comprometida con satisfacer las necesidades de su estudiantes, basada en la Internet de alta velocidad, dispositivos móviles, plataformas tecnológicas y aplicaciones digitales, que facilitan el aprendizaje personalizado en cualquier momento, en cualquier lugar y cambian los roles transmisivos de los docentes, considerando la integración de múltiples modalidades para la diversidad social como la educación presencial, en línea, híbrida, móvil, remota, inmersiva.

\subsection{La innovación en la educación superior y en el contexto turístico}

La Organización Mundial del Turismo ha indicado que "El brote mundial de COVID-19 ha llevado al mundo a su paralización, y el turismo ha sido el más afectado de todos los grandes sectores económicos, en un marco de incertidumbre exacerbada, contar con información actualizada y fiable es más importante que nunca, tanto para los turistas como para el sector" (UNWTO, 2020). El proceso descripto ha llevado a una abrupta contracción de la actividad turística en el mundo. Esto no es más que un nuevo desafío para una actividad económica que, ya ha demostrado, tiene como cualidad la de superar las crisis económicas locales y/o globales recuperándose de forma acelerada apenas las condiciones cambian. Pero esta vez la contingencia es internacional, signada por la pandemia sanitaria global, que equipara a todos los destinos en la necesidad de tomar decisiones proactivas $y$ superadoras. El COVID-19 ha puesto sobre la mesa dos cuestiones que modifican el día a día de la actividad turística: la salud y la seguridad, no solo personal e individual sino también de la comunidad del destino turístico, de los lugares de tránsito y del lugar de partida.

La innovación es el motor que llevará al turismo a su recuperación, buscando soluciones sostenidas por datos serios, concretos y fiables que brinden tranquilidad a los viajeros. La actividad turística ha demostrado frente a otras situaciones críticas, crisis económicas internacionales; catástrofes naturales que destruyen destinos; atentados terroristas; su flexibilidad y elasticidad encontrando opciones y oportunidades que la "reinventan". El turismo es una actividad resiliente, los actores del turismo son proactivos; van hacia las oportunidades y se adaptan a nuevos escenarios.

El reconocimiento de la educación como una institución social compleja exige de renovadas ideas sobre cómo gobernar los sistemas educativos (Burns y Köster, 2016). Es por ello, que las múltiples formas de gobernanza de los sistemas educativos, así como sobre sus posibilidades y límites deben proporcionar una mejora de la educación a nivel superior y la nuevas tendencias del sector turístico que se ha suscitado en este año 2020, y que se fundamenta en contar con un sistema educativo que ante la industria 4.0 requiera de profundas reflexiones, más allá de lo vocacional (Tsaih y Hsu, 2018), o desde el diseño y trascendencia de la experiencia (Qurashi y Sharpley, 2018), máxime cuando la literatura muestra que desde toda 
perspectiva de gestión turística, en los programas de estudio 0 en el mercado laboral o profesional es necesario avanzar hacia el turismo innovador e inteligente mediado por tecnología adoptados en la sostenibilidad y los valores (Lim, Mostafa y Park, 2017) y de manera independiente para cada una de las perspectivas que tiene el sector (Ghaderi, Hatamifar y Henderson, 2018; Koo, Shin, Kim, Kim y Chung, 2013), aun cuando existen algunos esfuerzos de integración que se realizan para análisis de la experiencia del turista (Kazak y Buchatskiy, 2018; Ballesteros, 2016; Rodríguez, 2018); adicionalmente, se debe migrar hacia la inteligencia, involucrando la gestión tecnológica del negocio a partir de las formas emergentes de las Tecnologías de Información y Comunicaciones, para brindar propuestas de valor conforme a las necesidades, no sólo de los consumidores, sino dando relevancia a todos los stakeholders, lo que implica pensar la industria y la región, desde la competencia, la colaboración, la comunidad y la academia (Dorcic, Komsic y Markovic 2019).

\section{DESARROLLO TEÓRICO:}

Esta investigación es de corte cualitativo, partiendo de la revisión de la literatura científica especializada en la materia. Para ello se realizó una revisión de fuentes secundarias de información, desarrollando este trabajo de investigación en tres vertientes. En primer lugar, se discute el rol de las ideas en la investigación sobre gobernanza de la educación superior, en el contexto del confinamiento y la cuarentena que han hecho emerger; en el segundo apartado, se enuncian las competencias que se identifican en base a los cambios disruptivos del siglo veintiuno y cómo se afrentarán en las disciplinas de turismo y la gastronomía y que implicarán al esquema de personas, empresas, gobierno, universidades, que podrían ser las competencias claves que se van a relacionar con el trabajo futuro, y finalmente, en el tercer apartado se establece la dinámica de los programas de estudio en estas áreas, su adopción a una tecnología de sostenibilidad y valores como formas de comunicación probablemente más acorde en una política pública hacia la gobernanza de manera no presencial que tienen mucho potencial para convertirse en fórmulas permanentes más ágiles y eficientes en la tecnología.

En este tenor, se puede establecer que la Universidad 4.0 es una descripción adecuada de las formas en que las universidades de todo el mundo deben responder a la nueva economía y las tendencias asociadas, como la disrupción digital y los mercados laborales que cambian radicalmente. Si las universidades se centran en mantenerse relevantes, deberían comenzar desde hoy a experimentar un cambio revolucionario a nivel organizacional, operacional, estructural, pedagógico, socio-cultural y cognitivo, y dará lugar a una gobernanza 4.0 la cual está totalmente alineada con la cuarta revolución industrial. Se habla de nuevas plataformas en combinación con la Internet de las para personalizar el aprendizaje de los alumnos. Esto obligará a los educadores tradicionales a ejecutar nuevos roles docentes que trasciendan la transmisión de contenidos declarativos, lo que impactarán en la vida cotidiana, las relaciones sociales, las experiencias laborales y de aprendizaje para toda la vida, debido a ello, es importante rediseñar los programas educativos principalmente en los ámbitos turísticos y gastronómicos a las nuevas circunstancias y tratando de incorporar las tecnologías emergentes, generando un cambio radical donde se debe acabar con las plantillas estancadas de personal, adaptar los procedimientos de promoción profesional, flexibilizar las relaciones con el entorno social, implantar planes estratégicos realistas, potenciar la interdisciplinariedad y la internacionalización de profesores-investigadores, alumnos y relaciones institucionales (Pulido, 2019).

Así mismo a nivel sistémico, se deben tener alternativas heutagógicas conjuntamente con la instrucción virtual lo que se pretende hacer en este momento es construir e invertir más en un ecosistema educativo robusto, no como una planificación de 
reemplazo o desplazamiento, sino como una forma de flexibilidad y adaptabilidad. Por lo tanto, esta nueva etapa se puede focalizar en un proceso de descentralización y evolución hacia un modelo de gobernanza más ágil, innovador y pertinente, con mayor horizontalidad y autonomías crecientes, preservando y fortaleciendo los fines y propósitos declarados por la Institución. Por lo tanto para definir una política de gobierno y gestión, en esta nueva etapa, es necesario recuperar los resultados favorables obtenidos en el devenir institucional e identificar las necesidades de ajuste en función de las limitantes y cambios de las condiciones de programación. En lo que refiere al modelo de gobernanza, la institución, atraviesa una etapa de transición entre la fase fundacional de la universidad basada en una estructura de conducción centralizada y vertical en materia de toma de decisiones, hacia una fase de consolidación y evolución institucional caracterizada por un enfoque emprendedor de gobernanza. El desarrollo de una IES y su sostenibilidad a largo plazo requiere de una disposición activa para adaptarse al contexto que la rodea y dar una respuesta eficaz a los desafíos que se presentan, tanto en lo relativo a las transformaciones estructurales que signan su macro ambiente y son transversales al conjunto de las actividades, como a las connotaciones específicas que dichas fuerzas generan en el sector, su entorno regional y local.

Con relación a lo anterior, se evidencia la necesidad de ir más allá de la funcionalidad de la tecnología y ver las perspectivas en la competencias clave para coadyuvar y fortalecer las aptitudes y actitudes, no solo de los estudiantes, sino de los actores involucrados en la triple hélice, con mirar a desarrollar destinos turísticos y territorios inteligentes, con el objetivo revisar desde los discursos de la academia, el sector y el Gobierno; las brechas y los factores clave de éxito en la implementación de nuevas tecnologías en el sector turístico. En este sentido, en Liburd, Nielsen y Heape (2019) encuentran una iniciativa de codiseño para el ámbito de turismo innovador desde la perspectiva de la gobernanza 4.0 en los programas educativos del sector, donde se agrupa al turismo y la gastronomía en la medida que de espacio a una variedad de oportunidades de aprendizaje que no se limitan a conferencias tradicionales o proyectos a corto plazo; por el contrario, abrir el currículo en espacios de aprendizaje que genere un cambio ontológico en la educación superior (Barnett, 2004:259). Defendiendo las ideas e ideales con otros, reproducir y revelar lo que se creemos que es importante. Esto implica un grado de intervención tanto en los estudiantes, los docentes, la institución, el gobierno y la sociedad.

Por lo tanto, la educación universitaria debe resolver una serie de nuevos desafíos como la adaptación a una nueva revolución tecnológica signada por la convergencia y transformación digital y de las comunicaciones.

Dado a lo anterior, es importante reflexionar para poder alcanzar estas metas y eso se suscita a replantear modelos educativos; así como de política pública en la educación, con el fin de ampliar los modelos actuales en una triple hélice: educación-formación y empleo, que se van a suscitar ahora con la Nueva Normalidad que se estableció a raíz del COVID-19, donde las instituciones educativas estarán desarrollando directrices sobre la garantía de calidad para el reconocimiento de las cualificaciones, en políticas para el desarrollo de las vías de aprendizaje en la educación superior, en una formación de alta calidad a fin de insertarse los estudiantes con el fin de continuar con una formación que genere una inserción laboral en el ámbito del área que se haya formado con el propósito de facilitar la comparación internacional y el reconocimiento de la educación a nivel mundial. En lo que compete a las disciplinas de la turismo y gastronomía, se debe contribuir a superar la visión objetual desde el proceso de teorización generado desde la Didáctica de las Ciencias Sociales; y por lo tanto romper el modelo económico imperante, basado en el consumo, donde el crecimiento ilimitado es la máxima que canibaliza los recursos naturales y culturales, y conlleva desigualdades sociales cada vez más pronunciadas. Una sociedad que busque 
solucionar estas diferencias por medio de medidas no sólo económicas sino integrales, debe actuar con inteligencia dado el grado de conocimiento existente, donde se involucra el desarrollo sostenible, y que con la gobernanza 4.0 en la educación superior; acentuar el desarrollo local se ha convertido en el nuevo activador de las políticas públicas en el margen del patrimonio, dimensionando en lo local o regional por encima de las nacionales, estatales y globales, derivado ahora por la pandemia que se suscitó en el primer trimestre del año 2020; despertar interés en una población apática, lograr cohesión social, con el fin de desencadenar actividades sostenibles y con ello mejorar los ingresos y la calidad de vida de la colectividad.

Se debe forjar un consenso para la recuperación y la innovación en los planes y programas de estudio en materia de turismo y gastronomía que garanticen mecanismos de refuerzo de la resiliencia de las IES ante futuras crisis, con especial atención al desarrollo de sus capacidades técnicas, tecnológicas y pedagógicas para usar apropiadamente metodologías no presenciales, así como sus capacidades de seguimiento de los estudiantes, en particular los más vulnerables, esta estrategia debería no solo fomentar la recuperación, allí donde los impactos de la crisis se hayan dejado sentir más, sino también el aprendizaje de las lecciones obtenidas, así como el fomento de la innovación, reflexionando acerca de la vigencia del modelo tradicional de educación superior hacia una reestructuración en un nuevo modelo de organización de los procesos de enseñanza aprendizaje híbrido, para mejorar la calidad del servicio y también la equidad, escalar la digitalización y el aprendizaje ubicuo, ya que muchas veces el recurso a tecnologías que exigen conectividad estable y con ancho de banda tiene un especial impacto en los sectores más vulnerables. El acceso a internet no siempre es posible y cuando lo es, la conectividad muchas veces tiene deficiencias. En este sentido, la virtualización tiene un especial impacto en los estudiantes en desventaja social y económica que solo recientemente se han beneficiado de los procesos de democratización y masificación en el ingreso a la educación superior.
Siendo la virtualización la principal herramienta educativa para sostener el funcionamiento de la educación, debe considerarse la enorme brecha digital. Su existencia debe ser reconocida, no para impedir la virtualización, sino para diseñar estrategias y mecanismos de apoyo que ayuden a combatirla con más intensidad aún.

Al mismo tiempo, en el marco de políticas públicas y con el apoyo de las industrias y de las compañías de telecomunicaciones, hay que promover la innovación, aprovechando el potencial de la digitalización para hacer realidad el principio del aprendizaje ubicuo o móvil, cuyas ventajas para la educación superior han sido objeto de múltiples estudios (Aljawarneh, 2019); lo que implica reconocer el potencial de los teléfonos celulares como herramientas de comunicación y de aprendizaje, así como de acompañamiento y seguimiento socioemocional de los estudiantes.

Hay muchas lecciones que aprender de la experiencia internacional acumulada con los MOOCS; desarrollar las capacidades de los docentes, ofreciendo incentivos y apoyos apropiados, para que el uso de soluciones y recursos tecnológicos que pueden mejorar la calidad de su trabajo se extienda; redefinir las modalidades contractuales de los docentes, teniendo en cuenta las implicaciones del teletrabajo académico y la docencia en línea que, sin duda, entrarán a formar parte de la nueva cotidianeidad de las IES.

Finalmente, derivado de esta investigación es importante promover la reflexión ética acerca de la integridad académica, la propiedad intelectual o el uso de datos privados en los programas virtuales. 


\section{REFERENCIAS:}

Duenas Ocampo, Sebastian, \& Perdomo-Ortiz, Jesús, \& Villa Castano, Lida Esperanza (2014). El concepto de consumo socialmente responsable y su medición. Una revisión de la literatura. Estudios Gerenciales, 30(132),287-300. [fecha de Consulta 4 de Septiembre de 2020]. ISSN: $0123-5923$.

Disponible en: https://www.redalyc.org/articulo. oa?id=212/21231380009

Elkington, J. y Hailes, J. (1989). The green consumer guide: From shampoo to champagne:

High-street shopping for a better environment. Londres: Gollancz.

González López, A. (2002). La preocupación por la calidad del medio ambiente. Un modelo cognitivo sobre la conducta ecológica. Tesis para optar al título de Doctor en Psicología social, Universidad Complutense de Madrid. Facultad de Psicología.

Madrid-España. Recuperado el 25 de abril de 2014,

de http://biblioteca.ucm.es/tesis/psi/ ucm-t26479.pdf.

Holahan, C. (2005). Psicología ambiental, un enfoque general. México: Limusa Editores.

Kotler Philip y Armstrong Gary (2013). Fundamentos de Marketing. México. Décimo Primera Edición. Pearson Educación. Recuperado de: https://issuu.com/issuesoto/docs/fundamento s_de_marketing_-_philip_k
Maloney, M. P. y Ward, M. P. (1973). Ecology: Let's Hear from the People. American Psychologist, 583-586.

Mejia Gil, M.C., \& Puerta Silva C.P. (2018). Consumo Responsable y configuración de ciudadanías proambientales. Región \& Cohesión, 8 (1), 77-106.

Newhouse, N. (1990). Implications of attitude and behavior research for environmental conservation. Journal of Environmental Education, 22(1), 26- 36.

Organización de las Naciones Unidas, ONU. (1992). Declaración de Río sobre el Medio Ambiente y el Desarrollo: Agenda 21. Recuperado de: https://www.un.org/spanish/esa/sust dev/agenda21/index.htm

Pascual del Riquelme Martínez, M., Peñalosa Otero, M., \& López Célis, D. (2015). consumo socialmente responsable en el mercado colombiano. Cuadernos Latinoamericanos De Administración, 11(20), 61- $\quad 70$. https://doi.org/10.18270/cuaderlam.v11i20.62 4

Peñalosa Otero, Mónica Eugenia, \& López Celis, Diana María. (2018). El estereotipo de los colombianos frente al consumo socialmente responsable. Pensamiento \& Gestión, (44), 243260. https://dx.doi.org/10.14482/peg e.44.10384 
Shaw, D. y Shiu, E. (2002). An assessment of ethical obligation and self-identity inethical consumer decision-making: A structural equation modelling approach. International Journal of Consumer Studies, 26(4), 286-293.

Roth, Eric. (2000). Psicología ambiental: interfase entre conducta y naturaleza. Revista

Ciencia y Cultura, (8), 63-78. Recuperado en 04 de septiembre de 2020, de http://www.scielo.org.bo/scielo.php? script=sci_arttext\&pid=S2077$33232000000200007 \& \operatorname{lng}=e s \& t \operatorname{lng}=$ es.

Vargas Martínez, Elva Esther, \& López Moreda, Laureano José, \& Martínez Cervantes, Rosa Susana (2014). LA DIMENSIÓN AMBIENTAL EN LAS PREFERENCIAS DE CONSUMO DEL TURISTA. CASO CANCÚN. Revista de Administração da Unimep, 12(2),42-58.[fecha de Consulta 4 de Septiembre de 2020]. ISSN: Disponible en: https://www.redalyc.org/articulo. oa?id=2737/273731798003

Webb, D. J., Mohr, L. A. y Harris, K. E. (2008). A reexamination of socially responsible consumption and its measurement. Journal of Business Research,61 (2), 91-98. 